\title{
An Extension of the $\tau$-Gauss Hypergeometric Functions and its Properties
}

\author{
Dinesh Kumar*
}

(Communicated by Nihal YILMAZ ÖZGÜR)

\begin{abstract}
Recently, an extension of the $\tau$-hypergeometric functions ${ }_{2} R_{1}^{\tau}(z)$ was given by Parmar [6]. The main object of this paper is to introduce an extension of the $\tau$-hypergeometric function ${ }_{3} R_{2}^{\tau}(z)$ and investigate its various properties such as integral representations, derivative formula, Mellin transform and fractional calculus operators. Some published results are the special cases of our main results.
\end{abstract}

Keywords: Generalized Gamma functions; Gauss's hypergeometric function; Generalized Gauss hypergeometric function; Extended $\tau$-hypergeometric function; Mellin transform; Fractional calculus operators.

AMS Subject Classification (2010): Primary: 33B20; 33C20; Secondary: 26A33; 33B15; 33C05.

*Corresponding author

\section{Introduction and Preliminaries}

Chaudhry and Zubair [1, 2] introduced extended gamma function, as follows:

$$
\Gamma_{p}(z)=\left\{\begin{array}{lr}
\int_{0}^{\infty} t^{z-1} \exp \left(-t-\frac{p}{t}\right) d t, & (\Re(p)>0 ; z \in \mathbb{C}) \\
\Gamma(z), & (\Re(z)>0 ; p=0)
\end{array} .\right.
$$

Srivastava et al. [9, p.487, eq.(15)] introduced the following family of generalized hypergeometric functions:

$$
{ }_{r} F_{s}\left[\begin{array}{c}
\left(\alpha_{1}, p\right), \alpha_{2}, \cdots, \alpha_{r} ; \\
\beta_{1}, \beta_{2}, \cdots, \beta_{s} ;
\end{array}\right]=\sum_{n=0}^{\infty} \frac{\left(\alpha_{1} ; p\right)_{n}\left(\alpha_{2}\right)_{n} \cdots\left(\alpha_{r}\right)_{n}}{\left(\beta_{1}\right)_{n}\left(\beta_{2}\right)_{n} \cdots\left(\beta_{s}\right)_{n}} \frac{z^{n}}{n !},
$$

where, $\left(\alpha_{1} ; p\right)_{n}$ is the generalized Pochhammer symbol [9, p.485, eq.(8)], defined as

$$
(\lambda ; p)_{\mu}=\left\{\begin{array}{cc}
\frac{\Gamma_{p}(\lambda+\mu)}{\Gamma(\lambda)}, & (\Re(p)>0 ; \lambda, \mu \in \mathbb{C}) \\
(\lambda)_{\mu}, & (\lambda, \mu \in \mathbb{C} ; p=0)
\end{array},\right.
$$

here, $(\lambda)_{\mu} \quad(\lambda, \mu \in \mathbb{C})$ denotes the Pochhammer symbol [10].

The integral representation of (1.1) is given by

$$
(\lambda ; p)_{\mu}=\frac{1}{\Gamma(\lambda)} \int_{0}^{\infty} t^{\lambda+\mu-1} \exp \left(-t-\frac{p}{t}\right) d t, \quad(\Re(p)>0, \Re(\lambda+\mu)>0 \text { when } p=0) .
$$

The $\tau$-hypergeometric function was investigated and studied by Virchenko et al. [11], defined by the following manner:

$$
{ }_{2} R_{1}^{\tau}(z)={ }_{2} R_{1}(a, b ; c ; \tau ; z)=\frac{\Gamma(c)}{\Gamma(b)} \sum_{n=0}^{\infty} \frac{(a)_{n} \Gamma(b+\tau n)}{\Gamma(c+\tau n)} \frac{z^{n}}{n !},
$$

Received : 05-February-2016, Accepted : 04-November-2016 
with $\tau>0 ;|z|<1 ; \Re(c)>\Re(b)>0$.

Integral representation of (1.2) given by [11, p.91, eq.(6)]:

$$
{ }_{2} R_{1}(a, b ; c ; \tau ; z)=\frac{1}{B(b, c-b)} \int_{0}^{1} t^{b-1}(1-t)^{c-b-1}\left(1-z t^{\tau}\right)^{-a} d t,
$$

with $\tau>0 ;|\arg (1-z)|<\pi ; \Re(c)>\Re(b)>0$.

When $\tau=1$ in (1.2) and (1.3) yields the representations of Gauss's hypergeometric function [7].

Very recently, Parmar [6, p.423, eq.(2.1)] introduced an extended $\tau$-hypergeometric function ${ }_{2} R_{1}^{\tau}(z)$, as follows:

$$
{ }_{2} R_{1}^{\tau}(z)={ }_{2} R_{1}^{\tau}((a, p), b ; c ; z)=\frac{\Gamma(c)}{\Gamma(b)} \sum_{n=0}^{\infty} \frac{(a ; p){ }_{n} \Gamma(b+\tau n)}{\Gamma(c+\tau n)} \frac{z^{n}}{n !},
$$

where, $a, b \in \mathbb{C}, c \in \mathbb{C} \backslash \mathbb{Z}_{0}^{-}, p \geq 0, \tau>0,|z|<1$, and $\Re(c)>\Re(b)>0$ when $p=0$, and its integral representation [6, p.423, eq.(3.1)]:

$$
{ }_{2} R_{1}^{\tau}((a, p), b ; c ; z)=\frac{1}{B(b, c-b)} \int_{0}^{1} t^{b-1}(1-t)^{c-b-1}{ }_{1} F_{0}\left[(a, p) ;-; z t^{\tau}\right] d t,
$$

with $\tau>0 ; \Re(p)>0 ; \Re(c)>\Re(b)>0$ when $p=0$.

\section{Extended $\tau$-Hypergeometric Function ${ }_{3} R_{2}^{\tau}(z)$}

Motivated mainly by investigations of the extended $\tau$-hypergeometric function ${ }_{2} R_{1}^{\tau}(z)$ defined by (1.2), we introduced the extended $\tau$-hypergeometric function ${ }_{3} R_{2}^{\tau}(z)$ as follows:

For $\lambda, a, b \in \mathbb{C}$ and $c, d \in \mathbb{C} \backslash \mathbb{Z}_{0}^{-}$, we have

$$
{ }_{3} R_{2}^{\tau}(z)={ }_{3} R_{2}^{\tau}((\lambda, p), a, b ; c ; d ; z)=\frac{\Gamma(c) \Gamma(d)}{\Gamma(a) \Gamma(b)} \sum_{n=0}^{\infty} \frac{(\lambda ; p)_{n} \Gamma(a+\tau n) \Gamma(b+\tau n)}{\Gamma(c+\tau n) \Gamma(d+\tau n)} \frac{z^{n}}{n !},
$$

where, $p \geq 0, \tau>0,|z|<1$, and $\Re(d)>\Re(a)>0, \Re(c)>\Re(b)>0$ when $p=0$.

\section{Special Cases:}

1. If we take $b=d$, then (2.1) reduces to the extended $\tau$-hypergeometric function ${ }_{2} R_{1}^{\tau}(z)$ given by Parmar $[6$, p.422, eq.(2.1)] as defined in (1.4).

2. If we put $b=d$ and set $\tau=1$, then (2.1) reduces to the extended Gauss hypergeometric function [9, p.487, eq.(17)] given by

$$
{ }_{2} F_{1}((\lambda, p), a ; c ; z)=\sum_{n=0}^{\infty} \frac{(\lambda ; p)_{n}(a)_{n}}{(c)_{n}} \frac{z^{n}}{n !}
$$

3. If we take $\tau=1$ and $p=0$ in (2.1), then it reduces to the classical Gauss's hypergeometric function as

$$
{ }_{3} F_{2}(\lambda, a, b ; c ; d ; z)=\sum_{n=0}^{\infty} \frac{(\lambda)_{n}(a)_{n}(b)_{n}}{(c)_{n}(d)_{n}} \frac{z^{n}}{n !} .
$$

4. If we put $b=d$ and set $\tau=1, p=0$ in (2.1), then it reduces to the classical Gauss's hypergeometric function as

$$
{ }_{2} F_{1}(a, b ; c ; z)=\sum_{n=0}^{\infty} \frac{(a)_{n}(b)_{n}}{(c)_{n}} \frac{z^{n}}{n !} .
$$




\section{Integral Representation and Derivative Formula}

In this section, we obtain integral representation and differential formula for ${ }_{3} R_{2}^{\tau}(z)$ as given in (2.1).

Theorem 1. The following integral representation for ${ }_{3} R_{2}^{\tau}(z)$ in (2.1) holds true:

$$
\begin{aligned}
& { }_{3} R_{2}^{\tau}((\lambda, p), a, b ; c ; d ; z) \\
& =\frac{1}{B(a, c-a)} \int_{0}^{1} t^{a-1}(1-t)^{c-a-1}{ }_{2} R_{1}^{\tau}\left[(\lambda, p), b, d ; z t^{\tau}\right] d t,
\end{aligned}
$$

where ${ }_{2} R_{1}^{\tau}(z)$ is given in (1.4), and $\tau>0 ; \Re(p)>0 ; \Re(d)>\Re(a)>0, \Re(c)>\Re(b)>0$ when $p=0$.

Proof. Using (2.1) and considering the following elementary identity for the Beta function:

$$
B(m, n)=\frac{\Gamma(m) \Gamma(n)}{\Gamma(m+n)}=\int_{0}^{1} t^{m-1}(1-t)^{n-1} d t,
$$

then we arrive at

$$
{ }_{3} R_{2}^{\tau}((\lambda, p), a, b ; c ; d ; z)=\frac{\Gamma(c)}{\Gamma(a) \Gamma(c-a)} \sum_{n=0}^{\infty} \frac{(\lambda ; p)_{n} \Gamma(b+\tau n)}{\Gamma(d+\tau n)} B(a+\tau n, c-a) \frac{z^{n}}{n !},
$$

now using (3.2), then we have

$$
=\frac{\Gamma(c)}{\Gamma(a) \Gamma(c-a)} \sum_{n=0}^{\infty} \frac{(\lambda ; p)_{n} \Gamma(b+\tau n)}{\Gamma(d+\tau n)} \int_{0}^{1} t^{a+\tau n-1}(1-t)^{c-a-1} \frac{z^{n}}{n !} d t .
$$

Next, interchanging the order of integration and summation which is permissible

$$
=\frac{\Gamma(c)}{\Gamma(a) \Gamma(c-a)} \int_{0}^{1} t^{a-1}(1-t)^{c-a-1}\left\{\frac{\Gamma(d)}{\Gamma(b)} \sum_{n=0}^{\infty} \frac{(\lambda ; p)_{n} \Gamma(b+\tau n)\left(z t^{\tau}\right)^{n}}{\Gamma(c+\tau n) n !}\right\} d t,
$$

by using (1.4), then we easily get the desired result in (3.1). This complete the proof of the Theorem 1.

If we put $b=d$, then (3.1) reduces to the known integral representation of the extended $\tau$-hypergeometric function ${ }_{2} R_{1}^{\tau}(z)$ given by Parmar [6, p.423, eq.(3.1)], as given in the following corollary:

\section{Corollary 1.1.}

$$
{ }_{2} R_{1}^{\tau}((\lambda, p), b ; c ; z)=\frac{1}{B(b, c-b)} \int_{0}^{1} t^{b-1}(1-t)^{c-b-1}{ }_{1} F_{0}\left[(\lambda, p) ;-; z t^{\tau}\right] d t,
$$

where $\Re(p)>0 ; \tau>0 ; \Re(c)>\Re(b)>0$ when $p=0$.

Remark 3.1. If we set $\tau=1$ in above corollary then we obtain the following result in terms of the extended hypergeometric function $[9$, p.488, eq.(24)]:

$$
{ }_{2} F_{1}((\lambda, p), b ; c ; z)=\frac{1}{B(b, c-b)} \int_{0}^{1} t^{b-1}(1-t)^{c-b-1}{ }_{1} F_{0}[(\lambda, p) ;-; z t] d t .
$$

Further, if we take $p=0$ in (3.3), then we arrive at the known integral representation of the classical Gauss's hypergeometric function [3, p.19, eq.(1.11.10)], as follows.

$$
{ }_{2} F_{1}(\lambda, b ; c ; z)=\frac{1}{B(b, c-b)} \int_{0}^{1} t^{b-1}(1-t)^{c-b-1}(1-z t)^{-\lambda} d t .
$$

Theorem 2. The following derivative formula for ${ }_{3} R_{2}^{\tau}(z)$ in (2.1) holds true:

$$
\left(\frac{d}{d z}\right)^{n}\left[z^{c-1}{ }_{3} R_{2}^{\tau}\left((\lambda, p), a, b ; c ; d ; \nu z^{\tau}\right)\right]=\frac{z^{c-n-1} \Gamma(c)}{\Gamma(c-n)}{ }_{3} R_{2}^{\tau}\left[(\lambda, p), a, b ; c-n ; d ; \nu z^{\tau}\right] .
$$


Proof. By using the series representation of ${ }_{3} R_{2}^{\tau}(z)$ as given in (2.1), and interchanging the order of differentiation and summation, then we arrive at the following:

$$
\begin{gathered}
\left(\frac{d}{d z}\right)^{n}\left[z^{c-1}{ }_{3} R_{2}^{\tau}\left((\lambda, p), a, b ; c ; d ; \nu z^{\tau}\right)\right] \\
=\frac{\Gamma(c) \Gamma(d)}{\Gamma(a) \Gamma(b)} \sum_{m=0}^{\infty} \frac{(\lambda ; p)_{m} \Gamma(a+\tau m) \Gamma(b+\tau m)}{\Gamma(c+\tau m) \Gamma(d+\tau m)} \frac{\nu^{m}}{m !}\left(\frac{d}{d z}\right)^{n}\left(z^{c+\tau m-1}\right),
\end{gathered}
$$

now, differentiating term by term under the sign of summation, we have

$$
\begin{aligned}
& =\frac{\Gamma(c) \Gamma(d)}{\Gamma(a) \Gamma(b)} \sum_{m=0}^{\infty} \frac{(\lambda ; p)_{m} \Gamma(a+\tau m) \Gamma(b+\tau m)}{\Gamma(c-n+\tau m) \Gamma(d+\tau m)} \frac{\nu^{m}}{m !} z^{c+\tau m-n-1} \\
& =z^{c-n-1} \frac{\Gamma(c) \Gamma(d)}{\Gamma(a) \Gamma(b)} \sum_{m=0}^{\infty} \frac{(\lambda ; p)_{m} \Gamma(a+\tau m) \Gamma(b+\tau m)}{\Gamma(c-n+\tau m) \Gamma(d+\tau m)} \frac{\left(\nu z^{\tau}\right)^{m}}{m !} .
\end{aligned}
$$

By using (2.1), we obtain the R.H.S. of (3.4) after little simplifications. This complete the proof.

\section{Mellin Transform of the Function ${ }_{3} R_{2}^{\tau}(z)$}

The Mellin transform of a function $f(x)$ is defined by

$$
\mathcal{M}[f(x) ; s]=F(s)=\int_{0}^{\infty} x^{s-1} f(x) d x, \quad(s \in \mathbb{C}),
$$

provided that the improper integral in (4.1) exists.

Theorem 3. The Mellin transform of the extended $\tau$-hypergeometric function ${ }_{3} R_{2}^{\tau}(z)$ defined in (2.1) is given by

$$
\mathcal{M}\left[{ }_{3} R_{2}^{\tau}((\lambda, p), a, b ; c ; d ; z) ; s\right]=\Gamma(s)(\lambda)_{s}{ }_{3} R_{2}^{\tau}(\lambda+s, a, b ; c ; d ; z),
$$

where $\Re(s)>0$ and $\Re(\lambda+s)>0$ when $p=0$.

Proof. Using (2.1) and applying Mellin transform, then we get

$$
\begin{gathered}
\mathcal{M}\left[_{3} R_{2}^{\tau}((\lambda, p), a, b ; c ; d ; z) ; s\right] \\
=\int_{0}^{\infty} p^{s-1}\left(\frac{\Gamma(c) \Gamma(d)}{\Gamma(a) \Gamma(b)} \sum_{n=0}^{\infty} \frac{(\lambda ; p)_{n} \Gamma(a+\tau n) \Gamma(b+\tau n)}{\Gamma(c+\tau n) \Gamma(d+\tau n)} \frac{z^{n}}{n !}\right) d p .
\end{gathered}
$$

Interchanging the order of integration and summation which is permissible, we have

$$
=\frac{\Gamma(c) \Gamma(d)}{\Gamma(a) \Gamma(b)} \sum_{n=0}^{\infty} \frac{\Gamma(a+\tau n) \Gamma(b+\tau n)}{\Gamma(c+\tau n) \Gamma(d+\tau n)} \frac{z^{n}}{n !} \frac{1}{\Gamma(\lambda)} \int_{0}^{\infty} p^{s-1} \Gamma_{p}(\lambda+n) d p .
$$

Now, using the result [2, p.16, eq.(1.110)] given by

$$
\int_{0}^{\infty} p^{s-1} \Gamma_{p}(\lambda+n) d p=\Gamma(\lambda+s+n) \Gamma(s), \quad(\Re(s)>0),
$$

then we arrive at the following:

$$
\begin{aligned}
\mathcal{M} & {\left[{ }_{3} R_{2}^{\tau}((\lambda, p), a, b ; c ; d ; z) ; s\right] } \\
& =\frac{\Gamma(s) \Gamma(c) \Gamma(d)}{\Gamma(\lambda) \Gamma(a) \Gamma(b)} \sum_{n=0}^{\infty} \frac{\Gamma(\lambda+s+n) \Gamma(a+\tau n) \Gamma(b+\tau n)}{\Gamma(c+\tau n) \Gamma(d+\tau n)} \frac{z^{n}}{n !} \\
& =\Gamma(s)(\lambda)_{s} \frac{\Gamma(c) \Gamma(d)}{\Gamma(a) \Gamma(b)} \sum_{n=0}^{\infty} \frac{(\lambda+s)_{n} \Gamma(a+\tau n) \Gamma(b+\tau n)}{\Gamma(c+\tau n) \Gamma(d+\tau n)} \frac{z^{n}}{n !} .
\end{aligned}
$$

By using the definition of extended $\tau$-hypergeometric function as given by (2.1), we obtain the desired result in (4.2). This complete the proof of the Theorem 3. 


\section{Fractional Calculus Approach}

The Riemann-Liouville left-sided fractional calculus operators $I_{a+}^{\alpha}$ and $D_{a+}^{\alpha}$ of order $\alpha$ are defined by [8]

$$
I_{a+}^{\alpha} f(x)={ }_{a} I_{x}^{\alpha} f(x)=\frac{1}{\Gamma(\alpha)} \int_{a}^{x}(x-t)^{\alpha-1} f(t) d t, \quad(x>a),
$$

is called the Riemann-Liouville left-sided fractional integral of order $\alpha$.

$$
D_{a+}^{\alpha} f(x)={ }_{a} D_{x}^{\alpha} f(x)=\frac{1}{\Gamma(n-\alpha)}\left(\frac{d}{d x}\right)^{n} \int_{a}^{x} \frac{f(t) d t}{(t-x)^{\alpha-n+1}}, \quad(n=[\alpha]+1),
$$

is called the left-sided Riemann-Liouville derivative of order $\alpha$.

In this section we consider the fractional differintegral operators containing extended $\tau$-hypergeometric function ${ }_{3} R_{2}^{\tau}(z)$ as in kernel.

Theorem 4. Let $\mu \in \mathbb{R}_{+}, \lambda, \alpha, a, b, c, d, \nu \in \mathbb{C}$ and $\Re(\alpha)>0, \Re(c)>0, \Re(\tau)>0$, then for $x>\mu$ the following result holds true:

$$
\begin{aligned}
& \left(I_{\mu+}^{\alpha}\left[(t-\mu)^{c-1}{ }_{3} R_{2}^{\tau}\left((\lambda, p), a, b ; c ; d ; \nu(t-\mu)^{\tau}\right)\right]\right)(x) \\
& =\frac{(x-\mu)^{c+\alpha-1} \Gamma(c)}{\Gamma(c+\alpha)}{ }_{3} R_{2}^{\tau}\left((\lambda, p), a, b ; c+\alpha ; d ; \nu(x-\mu)^{\tau}\right) .
\end{aligned}
$$

Proof. By using the series representation of extended $\tau$-hypergeometric function ${ }_{3} R_{2}^{\tau}(z)$ as given by (2.1) and interchanging the order of integration and summation, we have

$$
\begin{aligned}
& \left(I_{\mu+}^{\alpha}\left[(t-\mu)^{c-1}{ }_{3} R_{2}^{\tau}\left((\lambda, p), a, b ; c ; d ; \nu(t-\mu)^{\tau}\right)\right]\right)(x) \\
& =\frac{\Gamma(c) \Gamma(d)}{\Gamma(a) \Gamma(b)} \sum_{n=0}^{\infty} \frac{(\lambda ; p)_{n} \Gamma(a+\tau n) \Gamma(b+\tau n)}{\Gamma(c+\tau n) \Gamma(d+\tau n)} \frac{\nu^{n}}{n !} I_{\mu+}^{\alpha}\left((t-\mu)^{c+\tau n-1}\right)(x),
\end{aligned}
$$

now for $x>\mu$, taking the following power function formula into account:

$$
I_{a+}^{\alpha}(x-a)^{\beta-1}=\frac{\Gamma(\beta)}{\Gamma(\alpha+\beta)}(x-a)^{\alpha+\beta-1}, \quad(\alpha, \beta \in \mathbb{C}, \Re(\alpha)>0, \Re(\beta)>0),
$$

then we have

$$
\begin{aligned}
& \left(I_{\mu+}^{\alpha}\left[(t-\mu)^{c-1}{ }_{3} R_{2}^{\tau}\left((\lambda, p), a, b ; c ; d ; \nu(t-\mu)^{\tau}\right)\right]\right)(x) \\
= & \frac{(x-\mu)^{c+\alpha-1} \Gamma(c)}{\Gamma(c+\alpha)} \frac{\Gamma(c+\alpha) \Gamma(d)}{\Gamma(a) \Gamma(b)} \sum_{n=0}^{\infty} \frac{(\lambda ; p)_{n} \Gamma(a+\tau n) \Gamma(b+\tau n)}{\Gamma(c+\alpha+\tau n) \Gamma(d+\tau n)} \frac{\left[\nu(x-\mu)^{\tau}\right]^{n}}{n !},
\end{aligned}
$$

next, by using the definition (2.1), we obtain the desired result in (5.1).

Theorem 5. Let $\mu \in \mathbb{R}_{+}, \lambda, a, b, c, d, \nu \in \mathbb{C}$ and $\alpha>0, \Re(c)>0, \Re(\tau)>0$, then for $x>\mu$ the following relation holds true:

$$
\begin{aligned}
& \left(D_{\mu+}^{\alpha}\left[(t-\mu)^{c-1}{ }_{3} R_{2}^{\tau}\left((\lambda, p), a, b ; c ; d ; \nu(t-\mu)^{\tau}\right)\right]\right)(x) \\
& =\frac{(x-\mu)^{c-\alpha-1} \Gamma(c)}{\Gamma(c-\alpha)}{ }_{3} R_{2}^{\tau}\left((\lambda, p), a, b ; c-\alpha ; d ; \nu(x-\mu)^{\tau}\right) .
\end{aligned}
$$

Proof. By using the series representation given by (2.1) and using the following relation

$$
\left(D_{\mu+}^{\alpha} f\right)(x)=\left(\frac{d}{d x}\right)^{n}\left(I_{\mu+}^{n-\alpha} f\right)(x), \quad(\alpha \in \mathbb{C}, \alpha>0 ; n=[\alpha]+1),
$$


then we have

$$
\begin{aligned}
\left(D_{\mu+}^{\alpha}\left[(t-\mu)^{c-1}{ }_{3} R_{2}^{\tau}\left((\lambda, p), a, b ; c ; d ; \nu(t-\mu)^{\tau}\right)\right]\right)(x) \\
=\left(\frac{d}{d x}\right)^{n}\left(I_{\mu+}^{n-\alpha}\left[(t-\mu)^{c-1}{ }_{3} R_{2}^{\tau}\left((\lambda, p), a, b ; c ; d ; \nu(t-\mu)^{\tau}\right)\right]\right)(x),
\end{aligned}
$$

by using (5.1) and setting $\alpha=n-\alpha$, then we arrive at the following:

$$
\begin{gathered}
\left(D_{\mu+}^{\alpha}\left[(t-\mu)^{c-1}{ }_{3} R_{2}^{\tau}\left((\lambda, p), a, b ; c ; d ; \nu(t-\mu)^{\tau}\right)\right]\right)(x) \\
=\left(\frac{d}{d x}\right)^{n}\left[\frac{(x-\mu)^{c+n-\alpha-1} \Gamma(c)}{\Gamma(c+n-\alpha)}{ }_{3} R_{2}^{\tau}\left((\lambda, p), a, b ; c+n-\alpha ; d ; \nu(x-\mu)^{\tau}\right)\right] .
\end{gathered}
$$

next, by using the result (3.4), we obtain the desired result in (5.2) after little simplification. This complete the proof of the Theorem 5 .

Remark 5.1. For the detail of fractional calculus operators the reader can refer the work (see, [4, 5]).

\section{Concluding Remarks}

In the present paper we derive a new extended $\tau$-hypergeometric function ${ }_{3} R_{2}^{\tau}(z)$. Our results motivated mainly by investigations of the $\tau$-hypergeometric function ${ }_{2} R_{1}^{\tau}(z)$ [11] and its extension [6]. We obtained certain integral representation, a derivative formula, Mellin transform and fractional calculus approach of this new extended $\tau$-hypergeometric function ${ }_{3} R_{2}^{\tau}(z)$. The provided results are new and have uniqueness identity in the literature. On account of the general nature of the extended $\tau$-hypergeometric function, a number of known results can easily be found as special cases of our main results.

Acknowledgments The author (Dinesh Kumar) would like to express his deep thanks to NBHM (National Board of Higher Mathematics), India, for granting a Post-Doctoral Fellowship (sanction no.2/40(37)/2014/R\&D-II/14131).

\section{References}

[1] Chaudhry, M.A. and Zubair, S.M., Generalized incomplete gamma functions with applications. J. Comput. Appl. Math. 55 (1994), 99-124.

[2] Chaudhry M.A. and Zubair, S.M., On a class of incomplete Gamma functions with applications. CRC Press (Chapman and Hall), Boca Raton, FL, 2002.

[3] Gasper, G. and Rahman, M., Basic Hypergeometric Series. Cambridge University Press, Cambridge, 1990.

[4] Kumar, D. and Kumar, S., Fractional Calculus of the Generalized Mittag-Leffler Type Function. International Scholarly Research Notices 2014 (2014), Article ID 907432, 6 pages.

[5] Kumar, D. and Saxena, R.K., Generalized fractional calculus of the $M$-Series involving $F_{3}$ hypergeometric function. Sohag J. Math. 2 (2015), no. 1, 17-22.

[6] Parmar, R.K., Extended $\tau$-Hypergeometric functions and associated properties. Computes rendus Mathematique 353 (2015), no. 5, 421-426

[7] Rainville, E.D., Special Functions. Macmillan Company, New York, 1960; Reprinted by Chelsea Publishing Company, Bronx, New York, 1971.

[8] Samko, S.G., Kilbas, A.A. and Marichev, O.I., Fractional Integrals and Derivatives, Theory and Applications. Gordon and Breach, Yverdon et alibi, 1993.

[9] Srivastava, H.M., Çetinkaya, A. and Onur Kıymaz, İ, A Certain generalized Pochhammer symbol and its applications to hypergeometric functions. Appl. Math. Comput. 226 (2014), 484-491. 
[10] Srivastava, H.M. and Karlsson, P.W., Multiple Gaussian Hypergeometric Series. Halsted Press (Ellis Horwood Limited, Chichester), John Wiley and Sons. New York, Chichester, Brisbane and Toronto, 1985.

[11] Virchenko, N., Kalla, S.L. and Al-Zamel, A., Some results on a generalized hypergeometric function. Integral Transforms and Special Functions, 12 (2001), no. 1, 89-100.

\section{Affiliations}

DINESH KUMAR

AdDRESS: Department of Mathematics \& Statistics Jai Narain Vyas University, Jodhpur - 342005, India E-MAIL: dinesh_dino03@yahoo.com 